\title{
Dependence of Vascular Damage on Higher Frequency Components in the Rat-tail Model
}

\author{
Shilpi GOENKA ${ }^{1 a}$, Srikara V. PEELUKHANA ${ }^{2 a}$, Jay KIM ${ }^{2}$, \\ Keith F. STRINGER ${ }^{3}$ and Rupak K. BANERJEE ${ }^{2 *}$ \\ ${ }^{1}$ School of Energy, Environmental, Biological and Medical Engineering, Materials Engineering Program, \\ University of Cincinnati, USA \\ ${ }^{2}$ School of Dynamic Systems, Mechanical Engineering Program, University of Cincinnati, USA \\ ${ }^{3}$ Department of Pathology, Cincinnati Children's Hospital Medical Centre, USA
}

Received May 25, 2012 and accepted March 1, 2013

Published online in J-STAGE March 22, 2013

\begin{abstract}
Hand-Arm Vibration Syndrome (HAVS) is caused by hand-transmitted vibration in industrial workers. Current ISO guidelines (ISO 5349) might underestimate vascular injury associated with range of vibration frequencies near resonance. A rat-tail model was used to investigate the effects of higher frequencies $>100 \mathrm{~Hz}$ on early vascular damage. 13 Male Sprague-Dawley rats $(250 \pm 15 \mathrm{gm})$ were used. Rat-tails were vibrated at $125 \mathrm{~Hz}$ and $250 \mathrm{~Hz}\left(49 \mathrm{~m} / \mathrm{s}^{2}\right)$ for $1 \mathrm{D}, 5 \mathrm{D}$ and 10D; $D=$ days $(4 \mathrm{~h} /$ day). Structural damage of the ventral artery was quantified by vacuole count using Toluidine blue staining whereas biochemical changes were assessed by nitrotyrosine (NT) staining. The results were analyzed using one-way repeated measures mixed-model ANOVA at $p<0.05$ level of significance. The structural damage increased at $125 \mathrm{~Hz}$ causing significant number of vacuoles (40.62 \pm 9.8$)$ compared to control group $(8.36 \pm 2.49)$ and reduced at $250 \mathrm{~Hz}(12.33 \pm 2.98)$ compared to control group $(8.36 \pm 2.49)$. However, the biochemical alterations (NT-signal) increased significantly for $125 \mathrm{~Hz}(143.35 \pm 5.8$ gray scale value, GSV) and for $250 \mathrm{~Hz}(155.8 \pm 7.35 \mathrm{GSV})$ compared to the control group $(101.7 \pm 4.18 \mathrm{GSV})$. Our results demonstrate that vascular damage in the form of structural and bio chemical disruption is significant at $125 \mathrm{~Hz}$ and $250 \mathrm{~Hz}$. Hence the current ISO guidelines might underestimate vascular damage at frequencies $>100 \mathrm{~Hz}$.
\end{abstract}

Key words: Hand-arm vibration syndrome, Resonance frequency, Structural damage, Vacuoles, Biochemical alterations, Nitrotyrosine

\section{Introduction}

Hand-Arm Vibration Syndrome (HAVS) is an occupational disorder comprising vascular, sensorineural and musculoskeletal disorders prevalent amongst industrial workers who use hand-held powered tools for prolonged

*To whom correspondence should be addressed. E-mail: Rupak.Banerjee@uc.edu

${ }^{a}$ Both authors have equally contributed to the work

(C)2013 National Institute of Occupational Safety and Health durations ${ }^{1)}$. A report by the National Institute for Occupational Safety and Health (NIOSH) estimates that about half of the 1.2-1.5 million US workers who use hand-held powered tools are at risk of developing $\mathrm{HAVS}^{2)}$. Workers diagnosed with HAVS have shown evidence of severe vascular damage with symptoms of cold-induced vasospasms, vibration white finger $(\mathrm{VWF})^{3)}$, reduced blood flow and reduced tactile sensitivity in the hands and fingers ${ }^{4}$.

It has been reported that the resonance frequency of the human finger tissue is in the range $100-350 \mathrm{~Hz}^{5)}$. In addition, most of the tools, such as grinders and chipping 
hammers, used in industrial workplace generate significant vibration components in the frequency range 100$400 \mathrm{~Hz}^{6}$. However, ISO standard 5349 (2001) has positive frequency-weighting for the range between $32.5 \mathrm{~Hz}$ and $100 \mathrm{~Hz}$. The validity of this weighting has been questioned by researchers ${ }^{7-9)}$, who suspect the risk of high frequency components on vascular injury. This ISO guideline with higher weighting to lower frequency range might lead to an under estimation of the risk of high frequency components.

Several earlier reports investigating vascular damage centered on the frequency of $60 \mathrm{~Hz}^{8,10-14)}$, whereas higher frequency components in the range between $125 \mathrm{~Hz}$ and $250 \mathrm{~Hz}$ have gained increasing attention only recently ${ }^{9,15,16)}$. The cellular events responsible for early pathological damage in vasculature are still unclear for the frequencies near resonance range $(125-250 \mathrm{~Hz})$, which seem to play a key role in eliciting degenerative changes in the vascular tissue.

The rat tail is a simple structure that contains a large ventral artery, veins, and nerves that can be easily studied. In addition, the vasculature in the tail dilates or constricts in response to changes in ambient and body temperature, regulating blood flow ${ }^{17)}$. This temperature-induced vasoconstriction and vasodilation also occurs in fingers. In addition, it has already been validated that the biodynamic response of the rat-tail is similar to the human finger tissue ${ }^{16)}$ and several recent reports exist, which have used rat-tail model to study vibration-induced finger injury ${ }^{8-11,15,18)}$. Thus, the rat-tail vibration injury model can be used to elucidate the early degenerative changes in the vasculature, manifested by structural and biochemical alterations, under the influence of higher frequency components of vibration.

Considering the aforementioned factors, in this study, we sought to investigate the cellular changes due to vibration at near resonance frequencies, using a rat-tail model of vibration injury. We hypothesized that vascular damage, manifested in the form of structural damage (cellular vacuolation) and biochemical alterations (nitrotyrosine (NT)-mediated oxidative injury) ${ }^{19,20)}$ is dependent on the resonance frequencies $(125 \mathrm{~Hz}$ and $250 \mathrm{~Hz})$. Hence, in the present study, our aim was to evaluate the effects of vibration frequency $(125 \mathrm{~Hz}, 250 \mathrm{~Hz})$ on vascular damage.

\section{Subjects and Methods}

Animal Groups. The animal protocol for this study was approved by the Institute for Animal Care and Usage Committee (IACUC) at University of Cincinnati (UC). All procedures were in compliance with the NIH Guide for the Care and Use of Laboratory Animals. Vibration experi-

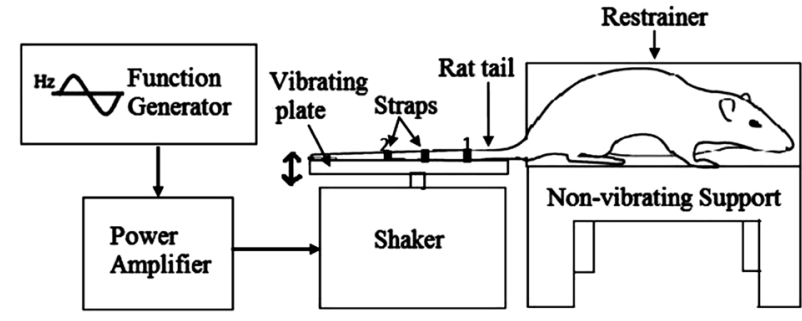

Fig. 1. Schematic representation of the rat-tail vibration apparatus. The rats were placed in Broome style restrainers with the tails strapped to a vibrating platform attached to the mechanical shaker. The tissue harvest sites on the tail are denoted by 1 and 2 .

ments were performed on non-anesthetized male SpragueDawley rats (250 \pm 15 gm, Harlan Laboratories, WI). The study consisted of a total of 13 rats. For the control group, 3 rats were utilized. 5 rats were assigned to $125 \mathrm{~Hz}$ group and 5 rats for the $250 \mathrm{~Hz}$ group. In the $125 \mathrm{~Hz}$ group, 1 rat-tail was vibrated for 1 day (D), 1 rat-tail for 5 days and 3 rat-tails for $10 \mathrm{D}$. Similar numbers of rats were used for $250 \mathrm{~Hz}$ group. The rats were housed in standard cages in a colony room at $25 \pm 1^{\circ} \mathrm{C}$ with a 12:12 light: dark cycle in the Laboratory for Animal Medicine and Services (LAMS) at UC and were provided the standard rodent diet and tap water.

Vibration Protocol. The vibration experiments were done at frequencies of $125 \mathrm{~Hz}$ and $250 \mathrm{~Hz}$, for duration of $4 \mathrm{~h} /$ day for 1D, 5D and 10D. The rats were placed in Broome style restrainers resting on a non-vibrating support and the tails were strapped to a platform, with a natural frequency higher than $600 \mathrm{~Hz}$. The rat-tails were secured to the platform using a surgical tape. The rats were monitored during the experiments in order to make sure the tail didn't bounce on/off the platform. This platform was connected to a mechanical shaker (model V203; Ling Dynamic Systems, Herts, UK) which generated vertical vibrations (Fig. 1). Control group rats remained in their respective cages and were not subjected to either the vibration stress or the constraint (straps) stress on the nonvibrating platform. The required sinusoidal frequency was produced by a function generator (Model HP 35660A; HP Inc, Palo Alto, CA) connected to a power amplifier (Model PA 25; Ling Dynamic Systems, Herts, UK). An accelerometer (Model U352B22; PCB group inc. Buffalo, $\mathrm{NY}$ ) was attached to the platform to measure and maintain the acceleration at a constant value during the experiment. The acceleration used for both the vibration frequencies was $49 \mathrm{~m} / \mathrm{s}^{2}$ instantaneous acceleration (unweighted). The amplitude was not kept constant and it might vary 
since acceleration and frequency were kept constant. After vibration exposure, rats were returned to their respective cages and housed in the colony room.

Animal Health Observations. The animals were monitored for health and behavior during the time of vibration exposure for all the days of vibration. We did not find any symptoms of chromodacryorrhea (porphyrin secretion) or change in body temperature or teeth grinding, similar to previously reported study by Yan et al., 2010 21). Also, the animals did not exhibit any signs of stress or aberrant behavior. Mostly, rats in all the groups remained calm in the restrainer during the vibration exposure.

Tissue Processing. After vibration exposure, rats were euthanized using carbon dioxide as per standard laboratory procedures followed by a secondary physical method of diaphragm puncture (approved by IACUC at LAMS, UC) and the ventral artery (after the tail was fixed for $24 \mathrm{~h}$ in $4 \%$ paraformaldehyde solution) was harvested from two different locations as shown in Fig. 1. The arteries were processed $24 \mathrm{~h}$ after the last vibration exposure. The tissue specimen from site 1 was fixed in $4 \%$ paraformaldehyde (for $24 \mathrm{~h}$ ) and cut into two separate artery specimens. One was paraffin-embedded, and the sections were stained with H\&E (hematoxylin and eosin) for routine histology. The other was treated with $30 \%$ sucrose in PBS for $72 \mathrm{~h}$ and frozen, and the sections were used for immunohistochemistry studies. The tissue specimen from site 2 (Fig. 1) was fixed in glutaraldehyde, post fixed in $1.3 \%$ osmium tetroxide and then plastic embedded using Epon (Resin LX112, Polysciences Inc.), to be further used for toluidine blue staining.

$H \& E$ Staining. Sections $(4 \mu \mathrm{m})$ were cut from paraffin blocks for H\&E staining and affixed onto glass slides. The slides were then deparaffinized using xylene and taken through a series of graded ethanol washes, stained with Hematoxylin and counterstained with Eosin. After cover-slipping, the slides were assessed at $\times 20$ objective magnification for changes in the morphology of artery sections and the smooth muscle cell (SMC) thickness, which was classified as the layer between the boundary of intima lining and the adventitia, was calculated using ImageJ software (National Institutes of Health, Bethesda, MD). For SMC thickness, four data points (taken at 3, 6, 9 and 12 o'clock) per section per rat were taken and averaged.

Vacuole Count. Plastic-embedded semi-thin artery sections $(1 \mu \mathrm{m})$ were used for toluidine blue staining and further for counting vacuoles $(2-12 \mu \mathrm{m} \text { in size })^{11,13)}$ under a light microscope. The artery sections were stained with $1 \%$ toluidine blue for two minutes, rinsed with deionized water and cover-slipped. The slides were then evaluated at $\times 40$ objective magnification and the total number of vacuoles was counted in the artery sections using ImageJ software. For every rat, vacuoles were counted in four randomly sampled sections.

Immunohistochemistry. Frozen tissues were cryostatsectioned at $6 \mu \mathrm{m}$, affixed to glass slides and then fixed in acetone $\left(-20^{\circ} \mathrm{C}\right)$. Next, the slides were dried in a laminar fume hood for ten minutes. Subsequently, all the glass slides were transferred into a humidifying chamber. Sections on the slides were rehydrated with $1 \mathrm{X}$ PBS solution and endogenous peroxidase was quenched by $3 \% \mathrm{H}_{2} \mathrm{O}_{2}$ solution (Ventana Inc., AZ). After these steps, the sections were washed with 1X PBS solution and then incubated with a mixture of $2 \%$ goat serum and $2 \%$ donkey serum ${ }^{22,23)}$ for 30 min to block nonspecific FC receptor binding by the primary and secondary antibodies. Subsequently, the sections were incubated with mouse anti-nitrotyrosine primary antibody (Santa Cruz Biotechnology, CA, 1:75, $4^{\circ} \mathrm{C}$ ) overnight. After washing steps were done using 1XPBS, the sections were incubated in biotinylated goat antimouse secondary antibody (Ventana, CA, 1:100, $30 \mathrm{~min}, 25^{\circ} \mathrm{C}$ ) and developed using the avidin biotinylated enzyme complex-diaminobenzidine (ABC-DAB) system (Vector Laboratories, CA). Sections were then coverslipped and photographed with a Spot RT Slider digital camera (Diagnostic Instruments, Sterling Heights, MI) mounted on a Nikon Eclipse E600 microscope. An intense brown coloration was indicative of a positive signal. Negative controls (which had the primary antibody omitted) were also performed to ensure that no nonspecific labeling was occurring via the secondary antibody. Additionally, as positive controls, peroxynitrite-treated sections (rich in nitro tyrosine) were used to evaluate the efficacy of the antibody staining procedure.

Quantification of NT immunostaining intensity was done in Adobe Photoshop ${ }^{\circledR}$ (Version CS2; Adobe Systems Inc. San Jose, CA) using six random samplings of $10 \times 10$ pixels each, based on an established densitometry method reported elsewhere ${ }^{24)}$. The digital images of the sections were shot at $\times 20$ objective magnification with a $10 \times$ ocular lens, inverted and zoomed in Photoshop (final magnification $400 \times$ ) and then six data points for the NT signal were taken from every section. In order to reduce sampling bias, 3-5 serial sections from every rat were investigated blindly by a pathologist. Three to five serial sections from every rat were assessed by a pathologist who was blinded to the group identities in order to reduce the observer $\operatorname{bias}^{25)}$. 
Statistical Analysis. Multiple sections were obtained from each rat. In order to account for the variability between the multiple sections (repeated measures) and for the variability in data between the rats, a one-way repeated measures mixed-model analysis of variance $(\text { ANOVA })^{26,27)}$ was used. A compound symmetry covariance structure was assumed for within the subject (repeated measures) and between the subject (random subject) effects. Group mean effects of frequency of vibration (pooled irrespective of the days of vibration; 1D, 5D, 10D) on structural damage (SMC thickness and vacuole count) and biochemical damage (NT signal intensity) were assessed.

For SMC thickness quantification 13 rats were used. There were 6 data points for the control group (from 3 rats), 11 data points for $125 \mathrm{~Hz}$ group (from 5 rats) and 10 data points for the $250 \mathrm{~Hz}$ group (from 5 rats). For vacuole count quantification, we had 5, 5 and 9 data point from the control ( 2 rats), $125 \mathrm{~Hz}$ (3 rats) and $250 \mathrm{~Hz}$ groups (3 rats), respectively. For NT signal intensity, we had 18 data points for the control group ( 2 rats), 54 data points for the $125 \mathrm{~Hz}$ group (5 rats) and 48 data points for the $250 \mathrm{~Hz}$ group (3 rats). In all the 190 analyses, animal was treated as the random effect while frequency was treated as fixed effect. The number of rats (subjects) is the $n$-value and each data point is considered an observation from each rat. Based on normal probability plots, Logarithmic transformation was performed on vacuole count data to obtain normal distribution and minimize the standard error ${ }^{28)}$. Data analysis was performed using SAS 9.1.3 (SAS Institute Inc, NC) with $p<0.05$ used as the probability level to accept statistical significance. All values are reported as mean $\pm \mathrm{SE}$.

Since our primary focus was on the effects of frequency on vascular damage, the results of the effects of duration of vibration on vascular damage were separated and discussed in Appendix 1.

\section{Results}

The qualitative and quantitative effects of various levels of vibration frequency, grouped over duration (day) effects of vibration on vascular damage were assessed by analyzing the structural damage (H\&E stained sections and vacuole count obtained from toluidine blue stained sections) and the biochemical alteration (NT immunoreactivity obtained by immunohistochemistry).

$H \& E$ Staining. The qualitative results obtained from H\&E staining on control and vibrated artery sections are shown in Fig. 2. Figure 2A is representative of the control group artery sections. Figure $2 \mathrm{~B}, 2 \mathrm{C}$ and $2 \mathrm{D}$ represent the artery sections of the $125 \mathrm{~Hz}$ group (vibrated for 1D, 5D and $10 \mathrm{D}$, respectively) while the Fig. 2E, $2 \mathrm{~F}$ and $2 \mathrm{G}$ represent artery sections of the $250 \mathrm{~Hz}$ group (vibrated for 1D, $5 \mathrm{D}$ and $10 \mathrm{D}$, respectively). On qualitative observation, the artery sections for the $125 \mathrm{~Hz}$ group showed many SMC vacuoles (Fig. 2B and 2C) compared to the control group (Fig. 2A) or the $250 \mathrm{~Hz}$ frequency group (Fig. 2E, 2F, and $2 \mathrm{G}$ ). In addition, in the same frequency group of $125 \mathrm{~Hz}$ (Fig. 2D) numerous internal elastic membrane (IEM) involutions were observed compared to other groups. No other vascular abnormalities or inflammation were observed in the artery sections stained with H\&E.

However, on further magnification of the artery sections for the $125 \mathrm{~Hz}$ group (Fig. 3), we observed rounding and elevation of the previously more flattened EC nuclei (Fig. 3B). This phenomenon, wherein the nuclei of elevated, peg-like columnar cells protrude into a lumen, known as hobnail cytomorphometry, was not resolvable at lower magnifications. Additionally, large vacuoles in SMC were also observed in the artery section belonging to the $125 \mathrm{~Hz}$ group (Fig. 3A).

The SMC thickness was quantified for the control and vibrated artery sections. The vibrated arteries showed vascular remodeling evidenced by changes in SMC thickness. The mean value of SMC thickness was higher for the $125 \mathrm{~Hz}$ group $(102.99 \pm 4.71 \mu \mathrm{m})$ as compared to either the control group $(86.41 \pm 6.23 \mu \mathrm{m})$ or the $250 \mathrm{~Hz}$ group $(97.36 \pm 4.85 \mu \mathrm{m})$. Near significance $(\mathrm{p}=0.05)$ was obtained for the $125 \mathrm{~Hz}$ frequency group, while the values for the $250 \mathrm{~Hz}$ frequency group remained insignificant.

In summary, routine histology using H\&E stain showed that $125 \mathrm{~Hz}$ vibration frequency caused (i) rounding and elevation of EC nuclei, (ii) vacuole formation and (iii) increased SMC thickness.

Toluidine Blue Staining. Semi-thin artery sections $(1 \mu \mathrm{m})$ were stained with toluidine blue and used for both qualitative study and quantitation of vacuoles in the endothelium and SMC of the control and vibrated artery. Fig. 4 shows the photomicrographs of toluidine-blue stained artery sections for control and vibrated rats. Fig. 4A represents the control group artery section. Fig. 4B, 4D and 4F represent artery images of the $125 \mathrm{~Hz}$ frequency group (after 1D, 5D and $10 \mathrm{D}$ vibration, respectively), whereas Fig. 4C, 4E and $4 \mathrm{~F}$ represent artery images of the $250 \mathrm{~Hz}$ group (after 1D, $5 \mathrm{D}$ and $10 \mathrm{D}$ of vibration, respectively). No vacuoles were observed in the control group (Fig. 4A). The artery sections in the $125 \mathrm{~Hz}$ group (Fig. 4B, 4D and 4F) had more SMC vacuoles compared to the $250 \mathrm{~Hz}$ group (Fig. 4C, 4E and $4 \mathrm{G}$ ). Presence of large number of SMC vacuoles was 


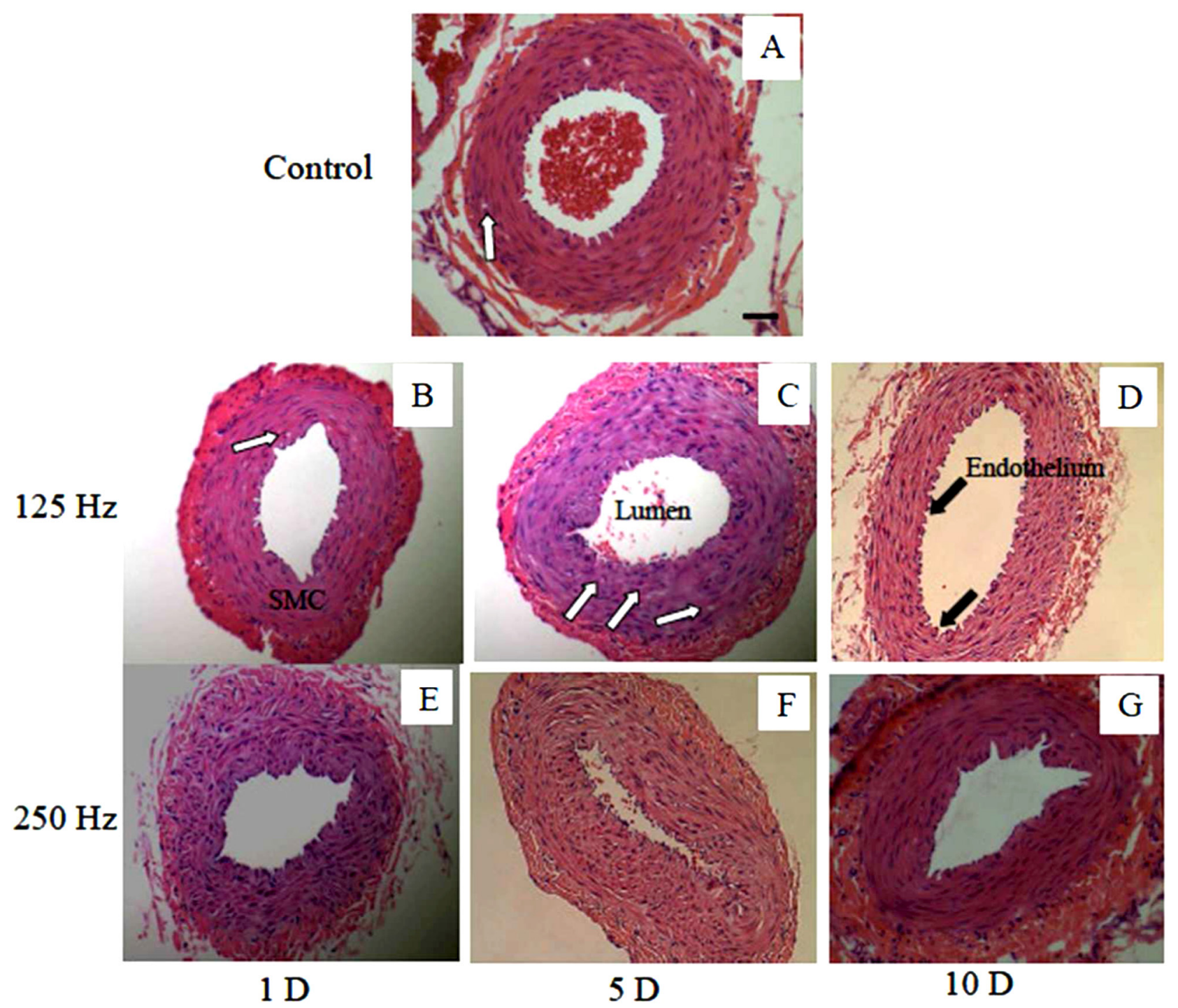

Fig. 2. Photomicrographs of the histological examination of control and vibrated artery sections using H\&E stain.

A. Non vibrated control, B. $125 \mathrm{~Hz}$ for 1D, C. $125 \mathrm{~Hz}$ for 5D, D. $125 \mathrm{~Hz}$ for 10D, E. $250 \mathrm{~Hz}$ for 1D, F. $250 \mathrm{~Hz}$ for 5D, G. $250 \mathrm{~Hz}$ for 10D. Very few SMC vacuoles (white arrows) are observed in the control artery (A) and the artery vibrated at $125 \mathrm{~Hz}$ for $1 \mathrm{D}$ (B) and 5D (C). 125 Hz at 10D (D) shows numerous IEM involutions (black arrows) compared to other groups. The high number of IEM involutions implies that EC are more pinched between them. Bar in A represents $50 \mu \mathrm{m}$ for all panels.

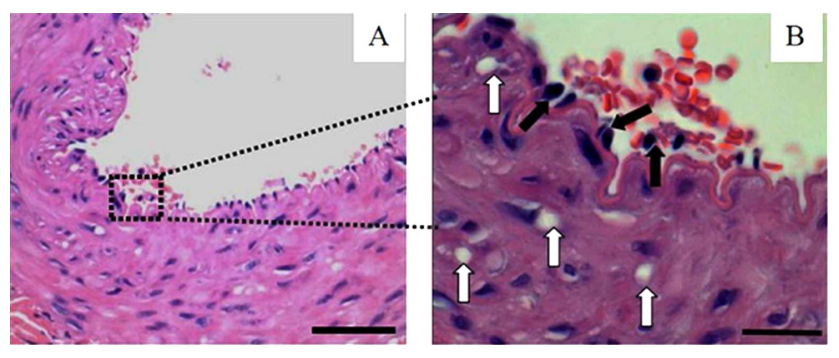

Fig. 3. Magnified H\&E image of artery vibrated at $125 \mathrm{~Hz}$ for $5 \mathrm{D}$.

A. $40 \times$, B. showing the corresponding image of the dotted section at magnification of $1000 \times$. Black arrows show the ECs with loss of normal flattened phenotype and with vertically oriented nuclei. Several vacuoles are visible in SMC (white arrows). Bar in 3A and 3B represent $50 \mu \mathrm{m}$ and $20 \mu \mathrm{m}$. similar to our previous qualitative observation of SMC vacuoles in $\mathrm{H} \& \mathrm{E}$ stained artery sections.

The extent of structural damage due to vibration was quantified by counting the total vacuoles in endothelium and SMC. The effects of vibration frequency on vacuole count in the artery are shown in Fig. 5. It was found that the increased vacuole count was statistically significant $(p<0.05)$ for $125 \mathrm{~Hz}$ group $(40.62 \pm 9.8)$, compared to the control group $(8.36 \pm 2.49)$. The number of vacuoles was significantly less $(p<0.05)$ for the $250 \mathrm{~Hz}$ group $(12.33 \pm$ $2.98)$ in comparison to the number of vacuoles observed for the $125 \mathrm{~Hz}$ group. However, no significance was 

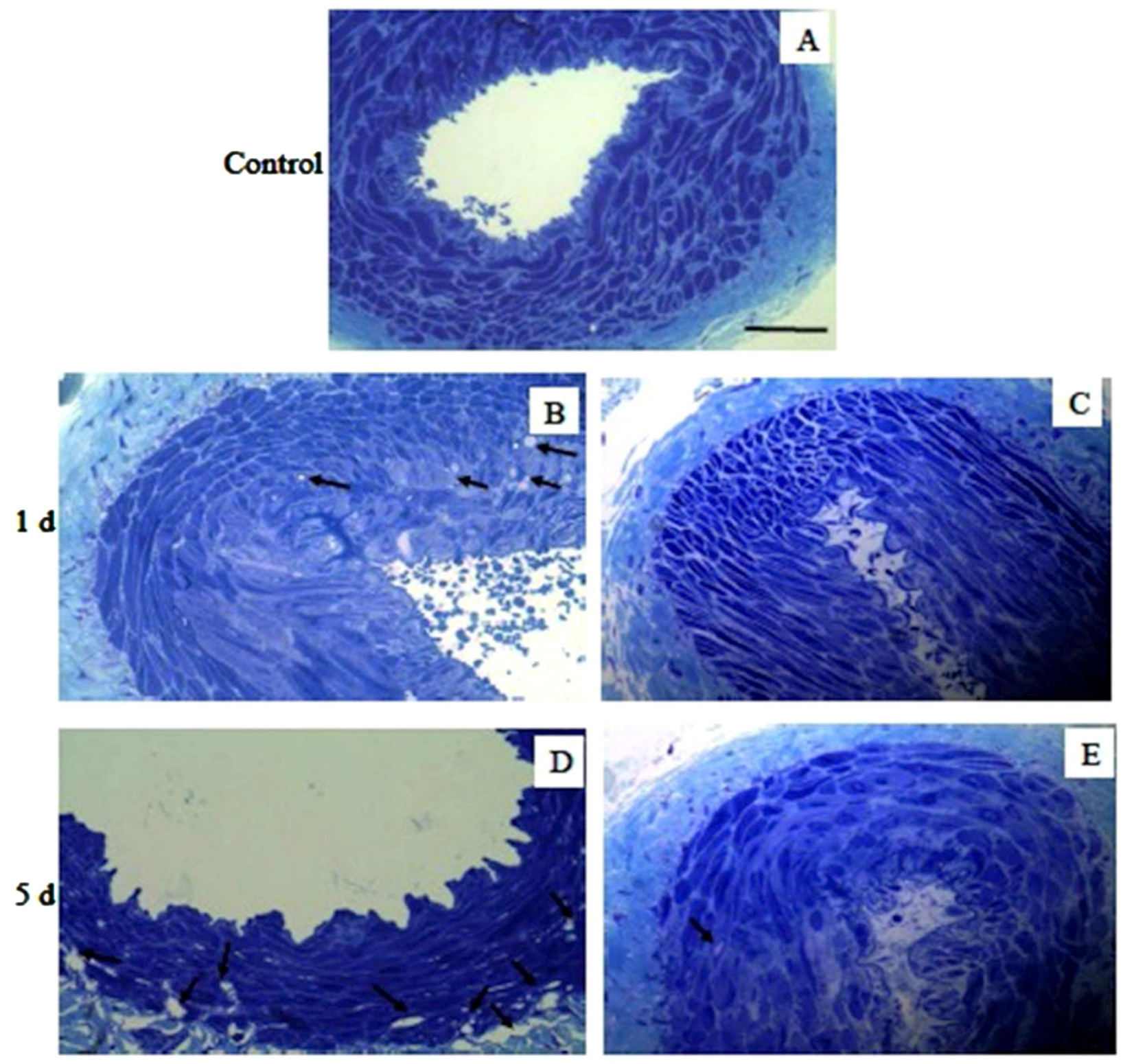

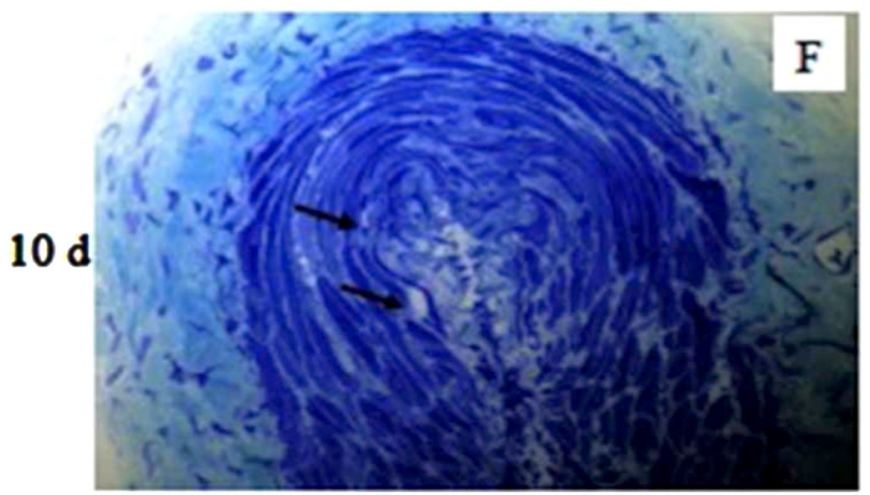

$125 \mathrm{~Hz}$

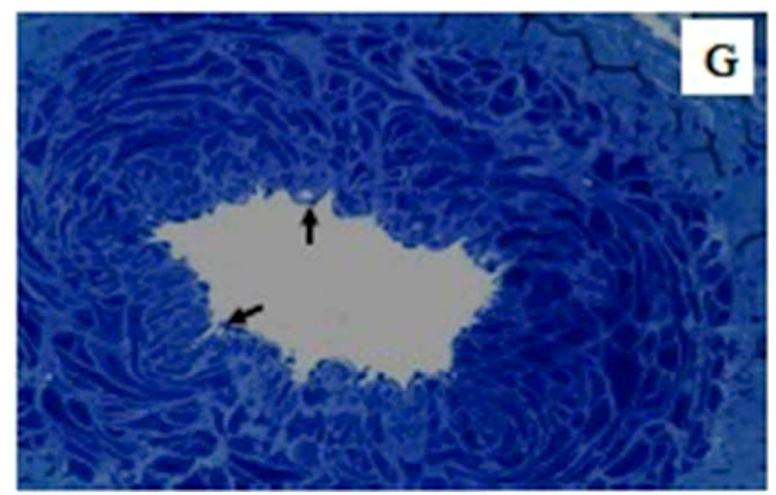

$250 \mathrm{~Hz}$

Fig. 4. Toluidine Blue stained sections of artery. Representative photomicrographs for:

A. Control, B. $125 \mathrm{~Hz}$ at 1D, C. $250 \mathrm{~Hz}$ at 1D, D. $125 \mathrm{~Hz}$ at 5D, E. $250 \mathrm{~Hz}$ at 5D, F. $125 \mathrm{~Hz}$ at 10D, G. $250 \mathrm{~Hz}$ at 10D. For the artery vibrated at $125 \mathrm{~Hz}$ for 5D (D), several swollen and larger vacuoles were observed (black arrows) towards the interface of adventitia and SMC, compared to vacuoles observed for other groups. Bar in A represents $50 \mu \mathrm{m}$ for all panels. 


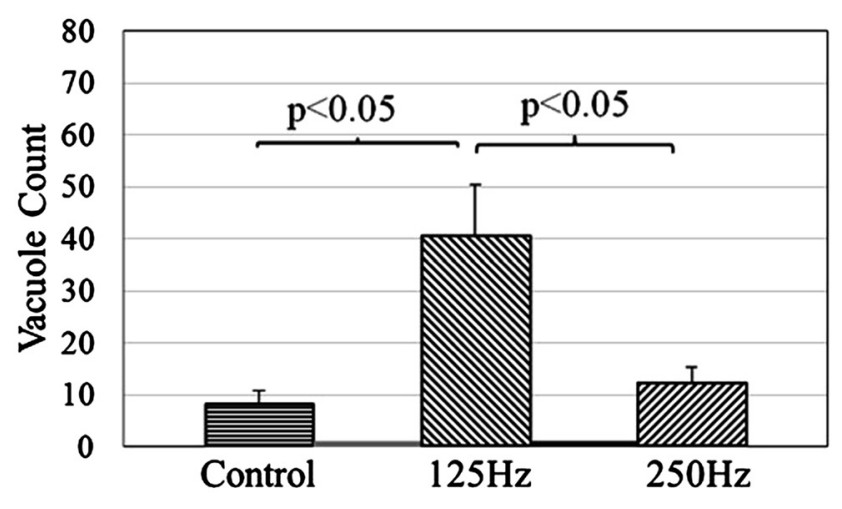

Fig. 5. Bar plot showing the main effects of frequency on vacuole count in the artery.

obtained between the vacuole counts of control group and $250 \mathrm{~Hz}$ group ( $p$-value $=0.33$ ). These results show that for $125 \mathrm{~Hz}$ frequency, vacuolization is increased in the artery, when compared with the control and the $250 \mathrm{~Hz}$ group.

Immunohistochemistry. Immunohistochemical staining was conducted on artery sections in order to assess the nitration of tyrosines in the protein, which is a marker of oxidative injury in the cell. Figure 6 illustrates the qualitative changes observed due to NT immunostaining for all the groups. Figure $6 \mathrm{~A}$ refers to the control group while Fig. 6B, 6D, and 6F represent the NT-stained artery sections of the $125 \mathrm{~Hz}$ group (vibrated for 1D, 5D, and 10D respectively). Similarly, Figure $6 \mathrm{C}, 6 \mathrm{E}$, and $6 \mathrm{G}$ represent NT-stained artery sections of the $250 \mathrm{~Hz}$ group (vibrated for $1 \mathrm{D}, 5 \mathrm{D}$, and $10 \mathrm{D}$, respectively). The presence of expressed NT protein was detected immunohistochemically in vibrated arteries (Fig. 6B-6G, brown signal involving the endothelium) and not in the non-vibrated control (Fig. 6A). Specifically, the $250 \mathrm{~Hz}$ group sections (Fig. 6C, 6E, and $6 \mathrm{G}$ ) showed higher contrast compared to the $125 \mathrm{~Hz}$ group (Fig. 6B, 6D, and 6F).

In order to carefully assess the cellular distribution of elevated NT protein, artery sections were assessed at higher magnification (using oil immersion and a 100× objective). Figure 7A and 7B show representative portions of NT-stained artery sections belonging to the $125 \mathrm{~Hz}$ and $250 \mathrm{~Hz}$ groups, respectively. The most intense NT signal is localized in the endothelium, and is pronounced in the artery sections of the $250 \mathrm{~Hz}$ group compared to the artery sections of the $125 \mathrm{~Hz}$ group.

Biochemical damage in the form of nitric oxidedependent oxidative injury was quantified based on the NT signal intensity. The effects of the frequency of vibration (averaged over days of vibration) on NT signal intensity in endothelium is shown in Fig. 8. The NT signal intensity in the endothelium was statistically significant $(p<0.05)$ for both the $125 \mathrm{~Hz}(143.35 \pm 5.8$ grayscale value, GSV) and $250 \mathrm{~Hz}(155.8 \pm 7.35 \mathrm{GSV})$ groups as compared to the control group $(101.7 \pm 4.18 \mathrm{GSV})$. However, the NT signal intensity between $125 \mathrm{~Hz}$ group and $250 \mathrm{~Hz}$ group remained statistically insignificant $(p$-value $=0.19)$. These results show that oxidative damage is significant at vibration frequencies of $125 \mathrm{~Hz}$ and $250 \mathrm{~Hz}$. However, for the $125 \mathrm{~Hz}$ group, the SMC exhibited very little (Fig. 6B) or no NT signal (Fig. 6D and 6F). Similarly, for the $250 \mathrm{~Hz}$ group, we found less staining in SMC compared to the more staining in endothelium (Fig. 6C, 6E, and 6G). These qualitative images show that the NT signal is present in vibrated artery sections and the signal appears darker in $250 \mathrm{~Hz}$ group compared to $125 \mathrm{~Hz}$ group, with the most intense signal being localized in the endothelium. The NT signal intensity was very low in the arterial smooth muscle compared to the higher levels in the endothelium. Thus, NT staining in SMC was not studied further.

Overall, these results demonstrate that the structural damage manifested by vacuoles was significant at $125 \mathrm{~Hz}$ compared to the control group. The decrease in vacuole counts was significant between the $125 \mathrm{~Hz}$ and $250 \mathrm{~Hz}$ groups. However, the biochemical alteration, assessed by NT signal intensity was significant for both the $125 \mathrm{~Hz}$ and $250 \mathrm{~Hz}$ groups, as compared to control. Although the NT signal intensity continued to increase with increasing vibration frequency (i.e. from 125 to $250 \mathrm{~Hz}$ ) the results were not statistically significant.

\section{Discussion}

We have studied vibration-induced vascular damage in the form of larger-scale structural damage (vacuole formation and cell loss easily visible using light microscopy) and also biochemical damage due to oxidative injury (detected using antibodies recognizing submicroscopic molecular alterations). Our results show that the structural damage increased at $125 \mathrm{~Hz}$ and attenuated at the $250 \mathrm{~Hz}$ frequency whereas the biochemical damage was significant at both the $125 \mathrm{~Hz}$ and $250 \mathrm{~Hz}$ vibration frequencies. The salient features of the study are listed below:

a. Simultaneous comparison of structural damage through vacuolization and biochemical alterations through nitrotyrosine staining at physiologically relevant frequencies of $125 \mathrm{~Hz}$ and $250 \mathrm{~Hz}$ at pertinent acceleration of $49 \mathrm{~m} / \mathrm{s}^{2}$.

b. Hobnail cytomorphology of EC observed in the H\&E and the blue toluidine stained sections. 

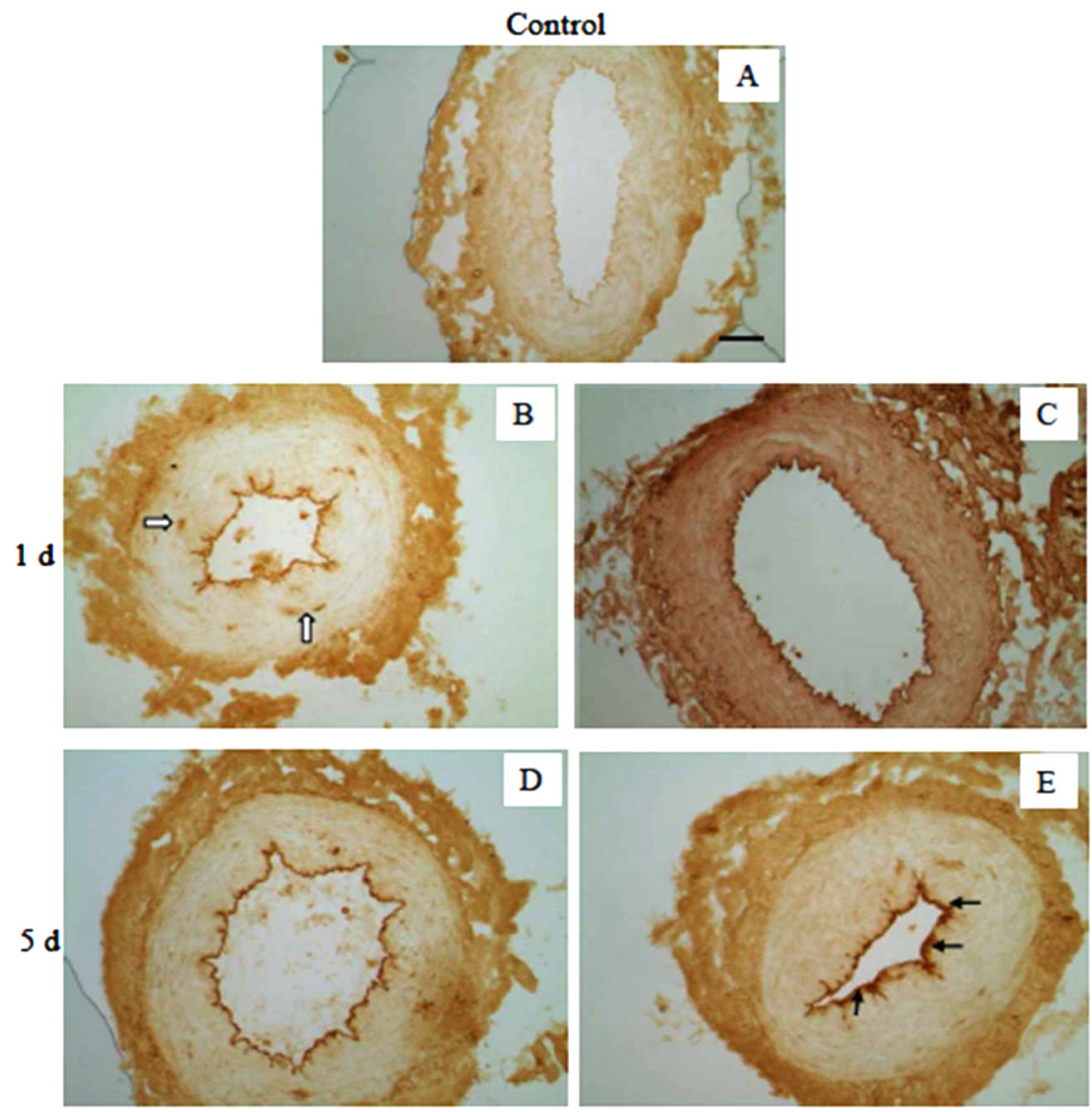

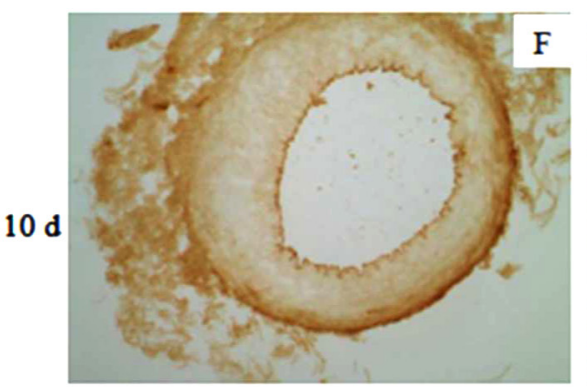

$125 \mathrm{~Hz}$

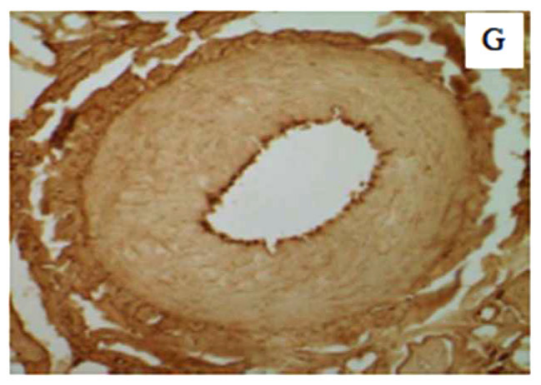

$250 \mathrm{~Hz}$

Fig. 6. Immunohistochemistry of NT in the artery. Representative photomicrographs for NT stained sections for:

A. Control, B. $125 \mathrm{~Hz}$ at 1D, C. $250 \mathrm{~Hz}$ at 1D, D. $125 \mathrm{~Hz}$ at 5D, E. $250 \mathrm{~Hz}$ at 5D, F. $125 \mathrm{~Hz}$ at 10D, G. $250 \mathrm{~Hz}$ at 10D. All the vibrated arteries showed NT signal (represented by dark brown color) in the endothelium, compared to control. For the artery vibrated at $250 \mathrm{~Hz}$ for $5 \mathrm{D}(\mathrm{E})$, the signal intensity for endothelium was high (arrows). Bar in A represents $50 \mu \mathrm{m}$ for all panels.

c. Differential NT staining observed in EC in comparison to SMC layer.

Structural Damage Mediated by increased SMC Thickness. We compared the effects of frequency and observed a slight increase in SMC thickness at $125 \mathrm{~Hz}$. SMC thickness is considered to be a useful indicator of vascular morphology changes and remodeling in the artery ${ }^{29)}$. An increased SMC thickness leads to a reduction in the lumen diameter resulting in vasoconstriction. Previous studies on changes in vibration-induced SMC thickness have either found no significant changes at $60 \mathrm{~Hz}$ vibration ${ }^{30}$ or found significant changes at frequency of $250 \mathrm{~Hz}^{9)}$. The near significant $p$-values obtained in our study suggest a slight increase in SMC thickness at $125 \mathrm{~Hz}$ frequency. Such increase in SMC thickness, not reported earlier, is in concurrence with the increased vacuole formation at the 


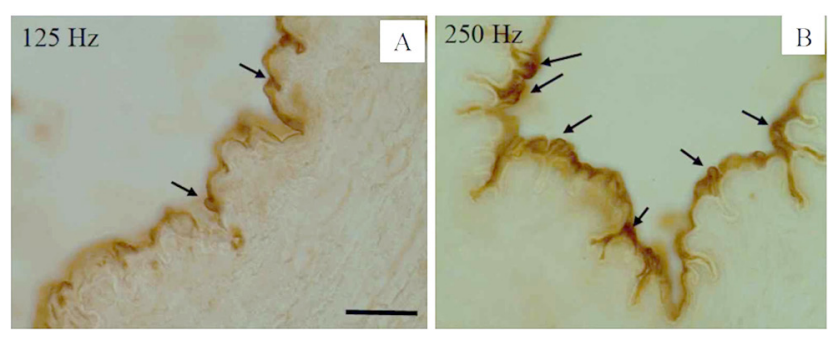

Fig. 7. Representative photomicrographs for NT stained sections for: A. $125 \mathrm{~Hz}$ at 5D, B. $250 \mathrm{~Hz}$ at 5D. The NT signal is clearly stronger for $250 \mathrm{~Hz}$ at $5 \mathrm{D}$ compared to $125 \mathrm{~Hz}$ at 5D. The endothelial cells in the 250 $\mathrm{Hz}$ at $5 \mathrm{D}$ group exhibited more extensive and intense signal (arrows) than those of the $125 \mathrm{~Hz}$ group. Bar represent $20 \mu \mathrm{m}$ for A and B.

same frequency.

Structural Damage mediated by Vacuole Formation. Our findings of vacuoles in H\&E stained sections are in contrast with a study in which no vacuoles were reported in arteries vibrated at $125 \mathrm{~Hz}^{31)}$. Vacuole formation in the artery is attributed to vibration-induced vasoconstriction ${ }^{11)}$. Moreover, it has been reported that the blood flow in the human finger is greatly reduced at the frequency of $125 \mathrm{~Hz}^{32,33)}$. Since reduced blood flow occurs due to enhanced vasoconstriction in the artery, it is possible that vasoconstriction is increased near this frequency. This suggests that the phenomena of vacuolation occur at a wide range of frequencies. It is possible that after the $125 \mathrm{~Hz}$ frequency, certain adaptive mechanism might be at play that prevents further structural damage as the frequency of vibration increases.

Biochemical Alteration Mediated by NT Formation. Previous studies on NT staining of the endothelium and SMC in vibrated arteries either reported NT staining in the SMC layer ${ }^{34)}$ or NT staining in both the endothelium and SMC layer ${ }^{13)}$. However, both of these studies were performed at vibration frequency of $60 \mathrm{~Hz}$ with vibration duration of $1 \mathrm{D}^{13)}$ or $1 \mathrm{D}$ and $3 \mathrm{D}^{34)}$.

Nitration reduces bioavailability of NO (nitric oxide) which is consumed in the chemical reaction. Our results of significant biochemical alteration (significant NT formation at $125 \mathrm{~Hz}$ and $250 \mathrm{~Hz}$ ) is in agreement with previous studies $^{9,35)}$ which reported that vascular NO concentration was reduced in arteries after vibration at $125 \mathrm{~Hz}^{35)}$. However, our observation of much lower intensity of staining in SMC compared to endothelial cells was somewhat different from observations in a recent study by Krajnak et al. ${ }^{9)}$. We observed strength of staining of NT in the EC that was not present in the SMC, implying a more active nitrosylation of peroxynitrite selective to the endothelium compared to SMC. We observed most intense staining

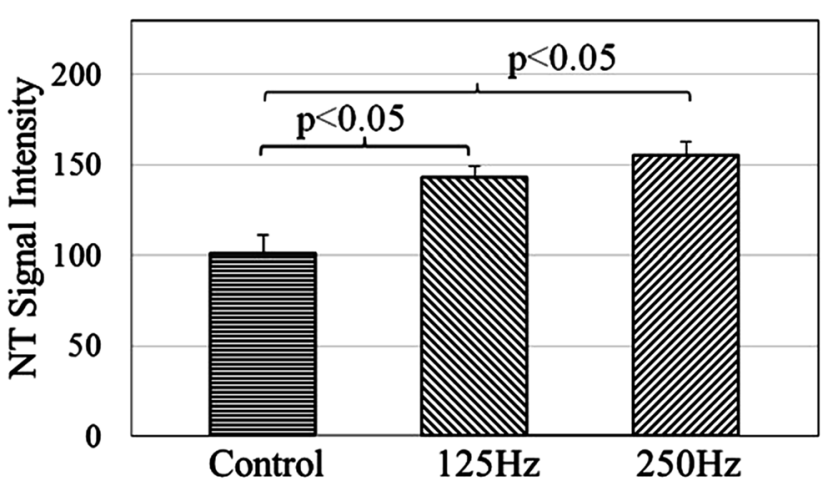

Fig. 8. Bar plot showing the main effects of vibration frequency on NT formation.

of NT in EC rather than SMC which might occur due to selective nitration of endothelial proteins by peroxynitrite, compared to SMC. Interestingly, this pattern of most intense signal in EC, that we observed has been reported elsewhere ${ }^{8,36)}$. Previous studies did not emphasize the region-specific response of oxidative damage under higher frequency vibrations. Thus, it is unclear whether the damage occurs in a heterogeneous manner in the endothelium and SMC. Nevertheless, it is well documented that the endothelium and the subendothelial space in arteries are more susceptible to tyrosine nitration and hence formation of $\mathrm{NT}^{37,38)}$. Since the cells in the endothelium and smooth muscle are phenotypically distinct, it is possible that their biochemical responses under vibration are varied. Further studies are warranted to elucidate the exact mechanisms causing this differential response of NT in endothelium and SMC.

The primary focus of this study is on the frequency effects; however, since the dependence of vascular damage on the duration of vibration is unclear, we also chose to do a preliminary study of the duration effects. We found that 5D and 10D time points presented a trend for both structural and biochemical alterations. However, we believe that this needs to be further validated with additional data in future studies.

Limitations. The sample size used in the present study is limited. Due to this, we consider this a preliminary study. However, we used multiple sections from each rat which allowed using the repeated measures data analysis. Based on these preliminary findings, further studies are planned to confirm findings with a larger sample size.

In our study, normal control group ${ }^{34)}$ has been used as opposed to rats sham-vibrated in restraints (restraint controls). The lack of restraint in a normal control group introduces additional bias. The rats in the normal control 
group haven't been exposed to either constraint or vibration stress. Therefore, the use of restraint controls could be important to assess any additional variables introduced into the analysis. The authors acknowledge this as a limitation and consider it to be a future study.

In this study, we investigated the effect of frequency dependence that is near resonance range on vibrationinduced vascular damage, under an instantaneous acceleration of $49 \mathrm{~m} / \mathrm{s}^{2}$. We chose these values since the vibration frequency responses in workers using hand held powered tools who are frequently exposed to these vibration parameters $\left(125 \mathrm{~Hz}, 250 \mathrm{~Hz}, 49 \mathrm{~m} / \mathrm{s}^{2}\right)$ can be better studied using rat-tail at these specific parameters ${ }^{16)}$. The effect of variable vibration amplitude was not considered in the present study. Staining of sections from two sites might have introduced uncontrolled variables and confounders. However, while location specific effects ${ }^{39)}$ might be important, no direct comparisons were made between the sections from these two disparate sites. The effect of different measurement location was not considered in the present study. Since HAVS is a complex disorder with interplay of vibration parameters of frequency, amplitude, acceleration and duration, future studies are warranted to give better assessment of vascular damage.

\section{Conclusions}

In summary, vascular damage, evaluated as structural damage occurred significantly at $125 \mathrm{~Hz}$ while biochemical alterations occurred significantly at both $125 \mathrm{~Hz}$ and $250 \mathrm{~Hz}$. These findings demonstrate that vascular damage is dependent on frequencies near resonance: $125 \mathrm{~Hz}$ and $250 \mathrm{~Hz}$. This leads to the conclusion that the current ISO guidelines might underestimate vascular damage at frequencies $>100 \mathrm{~Hz}$.

\section{Acknowledgements}

We would like to acknowledge the statistical guidance and advice provided by Dr. Paul Succop, Research Professor, Biostatistics, Department of Environmental Health, University of Cincinnati. We would also like to thank Dr. Amit Bhattacharya, Professor, Department of Environmental Health, for his thoughtful and timely advice during the course of this research. This work was supported by NIOSH-PRP grant \#: T42/OH008432-05. The authors are grateful to Dr. Randall Allemang, Director of the Structural Dynamics Research Laboratory, for granting access to the vibration equipment.

\section{References}

1) Heaver C, Goonetilleke KS, Ferguson H, Shiralkar S (2011) Hand-arm vibration syndrome: a common occupational hazard in industrialized countries. J Hand Surg Eur Vol 36, 354-63. [Medline] [CrossRef]

2) Bernard B, Nelson N, Estill CF, Fine L (1998) The NIOSH review of hand-arm vibration syndrome: vigilance is crucial. National Institute of Occupational Safety and Health. J Occup Environ Med 40, 780-5. [Medline] [CrossRef]

3) Stoyneva Z, Lyapina M, Tzvetkov D, Vodenicharov E (2003) Current pathophysiological views on vibration-induced Raynaud's phenomenon. Cardiovasc Res 57, 615-24. [Medline] [CrossRef]

4) Takeuchi T, Futatsuka M, Imanishi H, Yamada S (1986) Pathological changes observed in the finger biopsy of patients with vibration-induced white finger. Scand J Work Environ Health 12, 280-3. [Medline] [CrossRef]

5) Dong RG, Schopper AW, McDowell TW, Welcome DE, Wu JZ, Smutz WP, Warren C, Rakheja S (2004) Vibration energy absorption (VEA) in human fingers-hand-arm system. Med Eng Phys 26, 483-92. [Medline] [CrossRef]

6) Kihlberg S, Attebrant M, Gemne G, Kjellberg A (1995) Acute effects of vibration from a chipping hammer and a grinder on the hand-arm system. Occup Environ Med 52, 731-7. [Medline] [CrossRef]

7) Govindaraju SR, Curry BD, Bain JLW, Riley DA (2008) Nerve damage occurs at a wide range of vibration frequencies. Int J Ind Ergon 38, 687-92. [CrossRef]

8) Curry BD, Govindaraju SR, Bain JL, Zhang LL, Yan JG, Matloub HS, Riley DA (2005) Evidence for frequencydependent arterial damage in vibrated rat tails. Anat Rec A Discov Mol Cell Evol Biol 284, 511-21. [Medline]

9) Krajnak K, Miller GR, Waugh S, Johnson C, Li S, Kashon ML (2010) Characterization of frequency-dependent responses of the vascular system to repetitive vibration. $\mathrm{J}$ Occup Environ Med 52, 584-94. [Medline] [CrossRef]

10) Curry BD, Bain JL, Yan JG, Zhang LL, Yamaguchi M, Matloub HS, Riley DA (2002) Vibration injury damages arterial endothelial cells. Muscle Nerve 25, 527-34. [Medline] [CrossRef]

11) Govindaraju SR, Bain JL, Eddinger TJ, Riley DA (2008) Vibration causes acute vascular injury in a two-step process: vasoconstriction and vacuole disruption. Anat Rec (Hoboken) 291, 999-1006. [Medline] [CrossRef]

12) Curry BD, Govindaraju SR, Bain JL, Zhang LL, Yan JG, Matloub HS, Riley DA (2005) Nifedipine pretreatment reduces vibration-induced vascular damage. Muscle Nerve 32, 639-46. [Medline] [CrossRef]

13) Govindaraju SR, Curry BD, Bain JL, Riley DA (2006) Comparison of continuous and intermittent vibration effects on rat-tail artery and nerve. Muscle Nerve 34, 197-204. [Medline] [CrossRef]

14) Govindaraju SR, Curry BD, Bain JL, Riley DA (2006) 
Effects of temperature on vibration-induced damage in nerves and arteries. Muscle Nerve 33, 415-23. [Medline] [CrossRef]

15) Hughes JM, Wirth O, Krajnak K, Miller R, Flavahan S, Berkowitz DE, Welcome D, Flavahan NA (2009) Increased oxidant activity mediates vascular dysfunction in vibration injury. J Pharmacol Exp Ther 328, 223-30. [Medline] [CrossRef]

16) Welcome DE, Krajnak K, Kashon ML, Dong RG (2008) An investigation on the biodynamic foundation of a rat tail vibration model. Proc Inst Mech Eng H 222, 1127-41. [Medline] [CrossRef]

17) Berry JJ, Montgomery LD, Williams BA (1984) Thermoregulatory responses of rats to varying environmental temperatures. Aviat Space Environ Med 55, 546-9. [Medline]

18) Govindaraju S, Bain JLW, Riley DA (2007) Vibration causes ischemia-reperfusion injury in the rat-tail artery. FASEB J 21, A1220.

19) Al-Mufti RA, Williamson RC, Mathie RT (1998) Increased nitric oxide activity in a rat model of acute pancreatitis. Gut 43, 564-70. [Medline] [CrossRef]

20) Curtis CD, Thorngren DL, Nardulli AM (2010) Immunohistochemical analysis of oxidative stress and DNA repair proteins in normal mammary and breast cancer tissues. BMC Cancer 10, 9. [Medline] [CrossRef]

21) Yan JGZL, Yan YH, Sanger JR, Jensen ES, Matloub HS (2010) Improved animal model for vibration injury study. Scand J Lab Anim Sci 37, 159-69.

22) Song K, Nam YJ, Luo X, Qi X, Tan W, Huang GN, Acharya A, Smith CL, Tallquist MD, Neilson EG, Hill JA, BasselDuby R, Olson EN (2012) Heart repair by reprogramming non-myocytes with cardiac transcription factors. Nature 485, 599-604. [Medline] [CrossRef]

23) Sicklick JK, Li YX, Melhem A, Schmelzer E, Zdanowicz M, Huang J, Caballero M, Fair JH, Ludlow JW, McClelland RE, Reid LM, Diehl AM (2006) Hedgehog signaling maintains resident hepatic progenitors throughout life. Am J Physiol Gastrointest Liver Physiol 290, G859-70. [Medline] [CrossRef]

24) Matkowskyj KA, Schonfeld D, Benya RV (2000) Quantitative immunohistochemistry by measuring cumulative signal strength using commercially available software photoshop and matlab. J Histochem Cytochem 48, 303-12. [Medline] [CrossRef]

25) Skov BG, Braendstrup O, Hirsch FR, Lauritzen AF, Nielsen HW, Skov T (1994) Are pathologists biased by clinical information?: a blinded cross-over study of the histopathological diagnosis of mesothelial tumours versus pulmonary adenocarcinoma. Lung Cancer 11, 365-72. [Medline] [CrossRef]

26) Devore JL (2004) Probability and Statistics for Engineering and the Sciences, 8th Ed., 687, Thomson Books/Cole, Toronto.

27) Wolfinger R, Chang M (Eds.) (1995) Comparing the $S_{A} S^{\circledR}$ GLM and MIXED Procedures for Repeated Measurements Analysis. SUGI Proceedings.

28) Kutner M, Nachtsheim CJ, Neter J, Li W (2005) Applied linear statistical models, 5th Ed., 1396, McGraw-Hill Irwin, Boston.

29) Takeuchi T, Imanishi H (1984) Histopathologic observations in finger biopsy from thirty patients with Raynaud's phenomenon of occupational origin. J Kumamoto Med Soc 58, 56-70.

30) Okada A, Inaba R, Furuno T (1987) Occurrence of intimal thickening of the peripheral arteries in response to local vibration. Br J Ind Med 44, 470-5. [Medline]

31) Krajnak K, Dong RG, Flavahan S, Welcome D, Flavahan NA (2006) Acute vibration increases \{alpha\}2C-adrenergic smooth muscle constriction and alters thermosensitivity of cutaneous arteries. J Appl Physiol 100, 1230-7. [Medline] [CrossRef]

32) Welsh CL (1980) The effect of vibration on digital blood flow. Br J Surg 67, 708-10. [Medline] [CrossRef]

33) Bovenzi M, Welsh AJ, Della Vedova A, Griffin MJ (2006) Acute effects of force and vibration on finger blood flow. Occup Environ Med 63, 84-91. [Medline] [CrossRef]

34) Rowe DJ, Yan JG, Zhang LL, Pritchard KA Jr, Kao DS, Matloub HS, Riley DA (2011) The preventive effects of apolipoprotein mimetic D-4F from vibration injuryexperiment in rats. Hand (N Y) 6, 64-70. [Medline] [CrossRef]

35) Krajnak K, Waugh S, Johnson C, Miller R, Kiedrowski M (2009) Vibration disrupts vascular function in a model of metabolic syndrome. Ind Health 47, 533-42. [Medline] [CrossRef]

36) Bian K, Davis K, Kuret J, Binder L, Murad F (1999) Nitrotyrosine formation with endotoxin-induced kidney injury detected by immunohistochemistry. Am J Physiol 277, F33-40. [Medline]

37) Beckmann JS, Ye YZ, Anderson PG, Chen J, Accavitti MA, Tarpey MM, White CR (1994) Extensive nitration of protein tyrosines in human atherosclerosis detected by immunohistochemistry. Biol Chem Hoppe Seyler 375, 81-8. [Medline] [CrossRef]

38) Hirabayashi H, Takizawa S, Fukuyama N, Nakazawa H, Shinohara Y (2000) Nitrotyrosine generation via inducible nitric oxide synthase in vascular wall in focal ischemiareperfusion. Brain Res 852, 319-25. [Medline] [CrossRef]

39) Pattnaik S, Banerjee R, Kim J (2012) Spatial resonance in a small artery excited by vibration input as a possible mechanism to cause hand-arm vascular disorders. J Sound Vibrat 331, 1951-60. [CrossRef] 


\section{APPENDIX 1}

\section{Effects of duration of vibration on vascular damage}

The dependence of vascular damage at various durations of vibration at frequencies near resonance range in a rat-tail model is still unclear. Therefore, in the present study, in addition to the effect of vibration frequency, we assessed the dependence of vascular damage in form of structural and biochemical damage, on duration of vibration (1D, 5D and 10D).

To this effect, the H\&E, vacuole count and NT signal intensity data obtained from the control, $125 \mathrm{~Hz}$ and $250 \mathrm{~Hz}$ groups was also compared across the number of days of vibration (1D, 5D and 10D) to assess the duration effect on vascular damage. One-way repeated measures mixed-model ANOVA was used to validate the main effect of days of vibration (grouped over all frequencies: $125 \mathrm{~Hz}$ and $250 \mathrm{~Hz}$ ) on structural damage (SMC thickness and vacuole count) and biochemical damage (NT signal intensity). For SMC thickness, there were 6 data points from control ( 2 rats), 6 data points from 1D ( 2 rats), 6 data points from 5D group ( 2 rats) and 9 data points from the 10D group (5 rats). Similarly, for the vacuole count, we had 5 data points from control (2 rats) and 6 data points each from the 1D (2 rats), 5D (2 rats) and 10D groups (2 rats). Lastly, for NT signal intensity, we had 18 data points from control (2 rats), 36 data points for 1D (2 rats), 36 data points for 5D (2 rats) and 36 data points for 10D groups (2 rats).

Structural Damage. The vacuole count in the artery was significant for $10 \mathrm{D}$ vibration groups $(8.17 \pm 1.17)$ as com- pared to the control group $(1.95 \pm 1.22)$. The increase in vacuole count was also significant between $5 \mathrm{D}(2.33 \pm 1.17)$ and $10 \mathrm{D}$ vibration groups $(8.17 \pm 1.17)$. Although, in the literature, there are studies which have documented vacuole formation in arteries vibrated for $1 \mathrm{D}^{6,8,11-13)}(60 \mathrm{~Hz})$, no study has yet investigated if vacuolation at various durations of vibration exposure. In addition, the mean values of SMC thickness were higher and statistically significant at $5 \mathrm{D}$ vibration $(108.10 \pm 6.96 \mu \mathrm{m})$ as compared to the control group $(86.42 \pm 6.20 \mu \mathrm{m})$. The other time points, namely, 1D $(95.35 \pm 6.96 \mu \mathrm{m})$ and 10D vibration $(98.96 \pm$ $4.61 \mu \mathrm{m})$ did not show significance for any pairwise comparison, though a near significance $p$ value $(=0.13)$ was obtained for 10D group in comparison to the control.

Biochemical Damage. The mean values of the quantified NT signal in endothelium was statistically significant for 1D (147.96 $\pm 9.84 \mathrm{GSV}), 5 \mathrm{D}(157.53 \pm 9.84)$ and 10D $(143.21 \pm 7.13 \mathrm{GSV})$ time points as compared to the control group (101.70 \pm 10.02 GSV). No other pairwise comparison was found significant. Previous studies ${ }^{9,13,14,34)}$ on NT formation and oxidative damage in vibrated arteries did not compare the NT expression across time points.

Results show that structural damage and biochemical damage show a time window. The structural damage manifested by vacuole count was significant for 10D vibration, while SMC thickness was significant for $5 \mathrm{D}$ vibration time point. However, biochemical damage was significant for all time points, but the values did not increase linearly. The structural and bio-chemical changes may have different time windows.

Table 1. Summary of data points for Nitro-tyrosine analysis

\begin{tabular}{|c|c|c|c|c|c|}
\hline S.No & Control & 1D & $5 \mathrm{D}$ & $10 \mathrm{D}$ & \\
\hline \multirow[t]{2}{*}{ Control } & 2 rats & & & & 2 rats \\
\hline & 18 points & & & & 18 points \\
\hline \multirow[t]{2}{*}{$125 \mathrm{~Hz}$} & & $1 \mathrm{rat}$ & $1 \mathrm{rat}$ & 3 rats & 5 rats, \\
\hline & & 18 points & 18 points & $\begin{array}{l}18 \text { points, } \\
6 \text { points each }\end{array}$ & 54 points \\
\hline \multirow[t]{2}{*}{$250 \mathrm{~Hz}$} & & 1 rat, & $1 \mathrm{rat}$ & 1rat & 3 rats, \\
\hline & & 18 points & 18 points & 12 points & 48 points \\
\hline \multirow{2}{*}{\multicolumn{2}{|c|}{ Total rats }} & 2 rats, & 2 rats, & 4 rats, & 120 points \\
\hline & & 36 points & 36 points & 30 points & \\
\hline
\end{tabular}




\section{APPENDIX 2}

\section{Statistical justification}

\section{A. Data points}

The study consisted of a total of 13 rats. For the control group, 3 rats were utilized. 5 rats were assigned to $125 \mathrm{~Hz}$ group and 5 rats for the $250 \mathrm{~Hz}$ group. In the $125 \mathrm{~Hz}$ group, 1 rat-tail was vibrated for 1 day, 1 rat-tail for 5days and 3 rats for 10 days. Similar numbers of rats were used for $250 \mathrm{~Hz}$ group. We also obtained multiple sections and readings from each rat. However, not all rat tail crosssections are perfect sections in order to be used for data analysis. In order to make sure that we had reliable data, only good cross-sections were used. In this process, we had to discard data from a few rats due to which our design has become unbalanced. Due to the limited budget and high staining costs, we have focused our study exclusively on the effect of vibration frequency.

In order to address any concern about the data analysis, we have provided detailed statistical analysis methods we followed. To validate our analysis, we consulted a biostatistician (acknowledged in the manuscript). An example of our analysis in the case of nitro-tyrosine (NT) results is provided below. In order to quantify the effect of vibration frequency on biochemical damage we had to discard data from 3 rats. This left us with a total of 10 rats for the data analysis. Multiple sections were obtained from each rat and each data point corresponds to a reading from the sections. A summary of the number of sections and rats used for the NT analysis is provided in the Table 1.
B. One- factor ANOVA with repeated measures and random factor

Before performing the one-way, repeated measures, mixed model (with rats as random factor) analysis presented in the paper we have performed two other types of analyses summarized in Table 2 presented below. Multiple data points were obtained from each rat. Also, there is a variation in data obtained from each rat, which includes the locations effects and also the variation between the rats since the data comes from different rats and each at different time points. To add to these, the design is unbalanced in nature. In order to accounts for all these variations, a mixed model, random effect, repeated measures analysis was suggested by the biostatistician. Please note that the repeated measures involved obtaining multiple data from a single rat at a single time point (Table 1). It didn't involve a time variation in single rat. A constant symmetry covariance structure was assumed between the repeated measures and for the variation between the rats. Therefore, we have performed an analysis in $\mathrm{SAS}^{\circledR}$ with rats (subjects) as random effect, one-way repeated measures, mixed model ANOVA with Tukey adjustment for mean comparisons. The results of this analysis were presented in the paper. A comparison with the other analyses is presented in Table 2 . The other analyses have given statistical significance for all the group-mean comparison as opposed to only a few significant comparisons obtained in our analysis (Table 2). Despite this, based on the biostatistician's recommendation, we have chosen to present the most conservative analysis in the paper.

Table 2. Summary of p-values from various analyses for NT staining

\begin{tabular}{lccr}
\hline \multicolumn{1}{c}{ S.No } & $\begin{array}{c}\text { One-way, no repeated } \\
\text { measures, time points } \\
\text { not pooled }\end{array}$ & $\begin{array}{c}\text { Two-way, repeated measure, } \\
\text { no random effect }\end{array}$ & $\begin{array}{r}\text { One way, repeated, random } \\
\text { factor ANOVA (presented } \\
\text { in paper) }\end{array}$ \\
\hline $\begin{array}{l}\text { Significant } \\
\text { comparisons }\end{array}$ & All the comparisons \\
$\begin{array}{l}\text { at } 0.05 \text { level of } \\
\text { significance }\end{array}$ & & $\begin{array}{l}\text { Frequency: All comparisons } \\
\text { Time points: All comparisons } \\
\text { Interactions: All comparisons with control }\end{array}$ & Frequency: Control-125Hz \\
& & & $\begin{array}{r}\text { Control -250Hz } \\
\text { Time points: Control-1D } \\
\text { Control-5D } \\
\text { Control-10D }\end{array}$ \\
\hline Non-significant & None & Time points: 1D-10D time points & Frequency: 125Hz-250Hz \\
& & & Time points: 1D-5D \\
& & & 1D-10D \\
& & & 5D-10D \\
\hline
\end{tabular}

